\section{Magnetic resonance imaging of changes in the brain of children cured of acute lymphoblastic leukemia}

\author{
Vladislav P. Bondarenko, ${ }^{1,2}$ \\ Galina V. Tereschenko, ${ }^{1}$ \\ Mikhail M. Andrianov, ${ }^{1}$ \\ Yulia V. Rumyantseva, ${ }^{1}$ \\ Alexander I. Karachunsky, ${ }^{1}$ \\ Vladimir N. Kasatkin, ${ }^{1}$ \\ Alexander F. Karelin, ${ }^{1}$ \\ Viktor N. Anisimov, ${ }^{1,3}$ \\ Elena V. Zhukovskaya, ${ }^{1}$ \\ Alexander G. Rumyantsev ${ }^{1}$ \\ ${ }^{1}$ Dmitry Rogachev National Medical \\ Research Center of Pediatric \\ Hematology, Oncology and Immunology, \\ Ministry of Healthcare, Moscow; \\ ${ }^{2}$ Clinical Hospital 17, Moscow; \\ ${ }^{3}$ Lomonosov Moscow State University, \\ Moscow, Russian Federation
}

\begin{abstract}
This study was aimed to systematize magnetic resonance imaging (MRI) presentation of toxic leukoencephalopathy, to find the correlation between method of central nervous system (CNS) leukemia prevention and changes on MRI, to find relationship between existence leukoencephalopathy on imaging and neurocognitive deficits in pediatric patients after anti-leukemic therapy. Brain MRI data of 48 children, who underwent a therapy course according to the ALL-MB intermediate risk protocol, was evaluated. In accordance with two arms of this protocol, they received either radiation therapy, or additional intrathecal administration of chemotherapeutic agents as a prevention of CNS leukemia. Also, neurocognitive tests were performed. According to the results of the performed investigation, $10(50 \%)$ out of 20 children, who received cranial irradiation and $18(66.6 \%)$ out of 27 patients, who received only intrathecal therapy demonstrated abnormal brain changes (leukoencephalopathy) according to MRI data. Leukoencephalopathy was mostly presented by diffuse zones and localized predominantly in the frontal and temporal lobes. There was no correlation between method of CNS prevention and the existence of leukoencephalopathy on MRI. The analysis of our data did not show significant differences in brain damage and severity of cognitive impairment depending on the type of prevention of CNS leukemia. Moreover,
\end{abstract}

in this study no statistical correlation was found between leukoencephalopathy on MRI and neurocognitive impairment according to clinical tests data. Further long-term prospective studies and examinations should be performed to assess late neurotoxic effects.

\section{Introduction}

CNS damage is one of the common complications of anticancer therapy in patients with ALL. Intrathecal chemotherapy and cranial irradiation are used as preventive measures for CNS leukemia development. These therapeutic options are used separately or in a combination depending on a particular therapy protocol. ${ }^{1}$

Side effects of intrathecal administration of chemotherapeutic drugs and systemic chemotherapy are determined by pharmacogenetics and the kinetics of cytostatic drugs. Leukoencephalopathy is a characteristic complication caused by the intrathecal administration of Methothrexate, Cisplatinand, Cytarabine and Carmustine. ${ }^{2}$ Toxic effects induced by Methothrexate are the most common in pediatric patients with leukemia. neurotoxicity can be divided into early and late leukoencephalopathy. Marked neurologic symptoms with minimal morphological changes on MRI images are typically registered during the acute phase of CNS damage. ${ }^{3}$ White matter changes seen on MRI in such cases can be reversible. ${ }^{4}$ Clinical presentation may vary from insignificant symptoms to significant neurological deficit. ${ }^{5}$ Late methothrexate-leukoencephalopathy develops more slowly and ultimately results in permanent focal neurological deficits. Periventricular and central white matter is involved primarily (signal intensification on T2-weighted MR images), while subcortical U-fibers remain relatively unaffected. ${ }^{6}$ As a rule, the corpus callosum and the anterior commissure remain unchanged. These changes may be unilateral, bilateral, or ultimately, alternating between the two over the course of the disease. Typically, changes in the white matter MRI signal disappear sometime after the end of therapy, but may remain for the rest of a patient's life and may be accompanied by secondary nonspecific changes (gliosis and moderate ventricular dilatation). ${ }^{7}$

Cranial irradiation side effects can be categorized into acute, early-delayed and late toxicity. Acute toxicity from cranial irradiation occurs within days or at most first few weeks of therapy. Clinically it is characterized by nausea, vomiting,
Correspondence: Vladislav P. Bondarenko, Dmitry Rogachev National Medical Research Center of Pediatric Hematology, Oncology and Immunology, Ministry of Healthcare, 1 Samory Mashela str., 117997, Moscow, Russian Federation.

Tel.: +79260613118.

E-mail: vlad-bondr@yandex.ru

Key words: magnetic resonance imaging, acute lymphoblastic leukemia, neuroimaging, neurotoxicity, rehabilitation.

Contributions: the authors contributed equally.

Conflicts of interest: the authors declare no potential conflicts of interest.

Funding: none.

Received for publication: 6 December 2018 Accepted for publication: 5 July 2019

This work is licensed under a Creative Commons Attribution-NonCommercial 4.0 International License (CC BY-NC 4.0).

(C) Copyright: the Author(s), 2019

Licensee PAGEPress, Italy

Hematology Reports 2019; 11:7946

doi:10.4081/hr.2019.7946

headaches and cerebral edema. These complications are usually self-limiting. Earlydelayed (subacute) effects occurs within 3 months after end of irradiation. The clinical symptoms are similar to the acute toxicity with headaches, fatigue, irritability, anorexia, nausea and vomiting. These two types of radiation toxicity are not frequent for patients with leukemia, because in this case the total radiation dose is wellfractioned. Late toxicity occurs after 90 days after irradiation and has various presentations and most of them are irreversible. Most common complications are leukoencephalopathy, vascular toxicity (microhemorrhages, moyamoya pattern, radiation induced vascular malformations, mineralizating microangiopathy) and secondary tumors (meningiomas, gliomas, sarcomas). Radiographic presentation of radiation leukoencephalopathy is quite the same as in patients after intrathecal chemotherapy with leukoencephalopathy. ${ }^{8,9}$ Even though the MR-appearance of chemo- and radiotherapy side effects on the adult brain has been known for quite a while, the detection of CNS damage in children in the setting of incomplete myelination and ongoing formation of neural connections remains a radiodiagnostic challenge. ${ }^{10}$ It is known that with 
an increase in the time, that has passed since the end of therapy, there is a tendency to an increase in the manifestations of neurotoxicity. ${ }^{11,12}$ For example, it was evaluated the risk factors for stroke in a big cohort of 5year survivors of childhood cancer who were followed up for a median time of 26 years, and it was proved the correlation between the incidence of stroke and received dose during cranial irradiation. ${ }^{13}$ Therefore, antitumor therapy has a longterm impact on CNS and it is very important to obtain data on the morphofunctional status of CNS to correctly understand children's neurocognitive and psychological impairments. In this case, brain MRI could be a good method to predict further neurocognitive deficits at the period of rehabilitation and follow-up. But the correlation between visualized changes on brain MRI and neurocognitive functions is poorly studied yet. ${ }^{14}$ In the context of increasing survivors' quality of life, the diagnosis and evaluation of post-therapeutic CNS damage and the development of rehabilitative and corresponding therapeutic measures are one of the top priorities in modern Pediatric Oncology. ${ }^{15}$

\section{Objectives}

The systematization of brain MRI changes in children who have completed therapy in accordance with the protocol including intrathecal chemotherapy or cranial irradiation as the method of prevention of CNS leukemia.

The correlation between the method of CNS prevention and the characteristics of MRI presentation of leukoencephalopathy.

This research is also an attempt to find out the correlation between early neurotoxic changes in brain MRI and neurocognitive functions impairments.

\section{Materials and Methods}

\section{Patients}

Clinical data were updated on 01.11.2017. This is an observational study without control groups. In the study, we analyzed brain MRI results in 48 patients with ALL from the intermediate-risk group aged 2 to 15 years. According to the ALLMB-2008 therapy protocol, all patients from the intermediate-risk group were randomized into two arms of CNS leukemia prevention therapy prior to the beginning of consolidation-II (Figure 1).

In one randomization arm (28 patients), as a means of CNS leukemia prevention, six additional intrathecally-administered doses of chemotherapeutic agents (Methotrexate, Cytarabine and Prednisolone) were performed, without any cranial irradiation (ARM-1). Another randomization arm (20 patients) involved additional cranial irradiation, with the total dose of 12 Gy (ARM-2). The radiation fields included retro-orbital regions, the base of the skull as well as the deep regions in the area of the middle cranial fossa. The daily dose must be $1 \mathrm{~Gy}$ on Day 1 and 1.7 Gy on the following days. Irradiation was performed 5 days a week until the appropriate cumulative dose is reached.

The clinical characteristics of these 48 patients reflected the whole group of patients included in ALL-MB-2008 protocol (Table 1). All patients included in the study were in remission according to the recent data. History taking, and medical documentation processing revealed no premorbid CNS illnesses. The mean age of children at the beginning of the therapy was 7 years old (varied from 2 to 15 ) and it was the same at the both groups of patients.

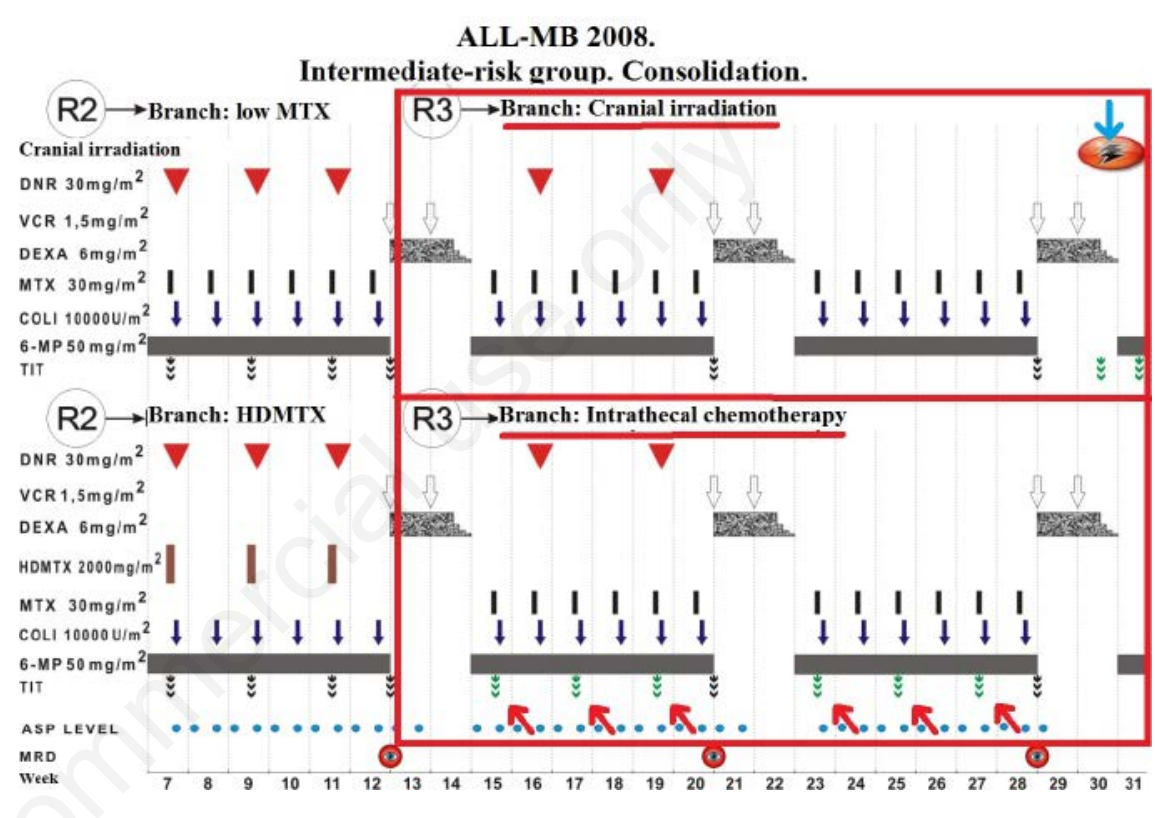

Figure 1. Consolidation phase of ALL-MB-2008. The intermediate-risk group.

Table 1. Initial characteristics of the patients.

\begin{tabular}{|c|c|c|c|c|}
\hline Characteristics & & MRI & & ed \\
\hline & $\mathbf{N}$ & $\%$ & $\mathrm{n}$ & $\%$ \\
\hline Sex & & & & \\
\hline Male & 20 & 41.7 & 554 & 58.8 \\
\hline Female & 27 & 56.3 & 389 & 41.2 \\
\hline Age & & & & \\
\hline$<10$ years & 36 & 75 & 711 & 75.4 \\
\hline $10-15$ years & 12 & 25 & 163 & 17.3 \\
\hline$>15$ years & 0 & 0 & 69 & 7.3 \\
\hline White blood cells, & & & & \\
\hline$<50$ & 36 & 75 & 655 & 69.5 \\
\hline$>50$ & 12 & 25 & 288 & 30.5 \\
\hline Spleen & & & & \\
\hline$<4 \mathrm{~cm}$ & 16 & 33.3 & 298 & 31.6 \\
\hline$>4 \mathrm{~cm}$ & 32 & 66.7 & 645 & 68.4 \\
\hline Immunophenotype & & & & \\
\hline Non-T-ALL & 33 & 68.8 & 685 & 72.6 \\
\hline T-ALL & 15 & 31.2 & 258 & 27.4 \\
\hline Total & 48 & & 943 & \\
\hline
\end{tabular}




\section{Imaging}

In 2014, after completion remission in consolidation phase, the patients underwent a course of rehabilitation at the Rehabilitation Research Centre "Russkoe Pole" of the "Dmitry Rogachev National Medical Research Center of Pediatric Hematology, Oncology and Immunology". That time the MRI study of brain was carried out with various timing after the completion of consolidation. MRI was performed on average 26 months after the end of the consolidation phase (varied from 9 days to 56 months; 21 months for cranial irradiation group and 31 months for intrathecal chemotherapy group).

MRI was performed on Philips Achieva 3T. The scanning protocol included T1 3D FRFSE, Axial T2 FLAIR, Axial T2 FRFSE, SWI, Axial DWI, Sagittal T2 FRFSE. No contrast enhancement was performed. During the analysis of the obtained data, the following parameters were assessed: the thickness and folding of the cortex of the cerebral hemispheres and cerebellum as well as the existence of foci therein; the presence or absence of white matter damage as well as its type (focal or diffuse).

\section{Neurocognitive tests}

Neurocognitive function in patients tested at the same time as the MRI was performed. Thy test system included tests of working memory, learning and executive function; visual, verbal and episodic memory; attention, information processing and reaction time; social and emotion recognition, decision making and response control. Comparative groups were children who received only intrathecal chemotherapy and did not receive prophylactic radiation. The following techniques were used to assess cognitive functions and behavior: CANTAB eclipse (Cambridge Cognition) hardware and software complex test battery, T. Achenbach questionnaire (Child Behavior Checklist, CBCL), M. Kovach Child Depression Inventory, CDI. ${ }^{16}$

\section{Statistics}

The estimation of the data was carried out by the methods of multiple regression analysis, assessed the complex effect of several factors on the dependent variables of interest. All statistical calculations were performed using the STATISTICA program (by StatSost).

\section{Results}

\section{Imaging}

According to the MRI, areas of leukoencephalopathy were detected in 10 $(50 \%)$ out of 20 children, who received radiotherapy (ARM-2). Focal changes in white matter of cerebral hemispheres were found in $8(80 \%)$ out of these 10 cases (Figure 2), whereas the combination of diffuse and focal areas was registered in 2 patients $(20 \%)(\mathrm{P}=0.095)$. In $3(30 \%)$ patients foci of leukoencephalopathy were localized just in one area (frontal or parietal) and two or more areas were involved in 7 patients $(70 \%)$.

As for patients, who received additional intrathecal injections of chemotherapeutic agents (ARM-1), MRI signs of leukoencephalopathy were found in 17 (66.6\%) out of 27 of them (one patient was excluded) $(\mathrm{P}=0.095)$. Focal involvement of the white matter of the cerebral hemispheres was detected in $12(70.6 \%)$ out of these children, diffuse involvement - 3 patients $(17.6 \%)$ and the combination of diffuse and focal areas was registered in 2 patients (11.8\%) (Figure 3). All results of MRI studies are shown in Table 2.

Thus, white matter changes in brain were registered in $10(50 \%)$ patients after combined-modality treatment (ARM-2) and in $17(66.6 \%)$ patients after course with extra intrathecal chemotherapy (ARM-1). A statistical analysis of the correlation the existence of white matter lesions and the method of CNS leukemia prevention (intrathecal chemo- or radiotherapy) was made. Base on the multiple regression model, there was no correlation between these factors $(\mathrm{P}<0.076)$.

Follow up of the patients' checked 2 of them with relapse. One patient, who received cranial irradiation, developed isolated testicular relapse. He was not excluding from the study group, as a repeat CSF examination did not reveal pathological cytosis and specific CNS involvement.

\section{Imaging of excluded patient}

It was detected that one patient with intrathecal chemotherapy had diffuse damage to the white matter of the cerebral hemispheres and the cerebellum as well as the damage to the cortex of the cerebral hemispheres, the pons and the cerebral peduncles. Apart from that, this patient had multi-
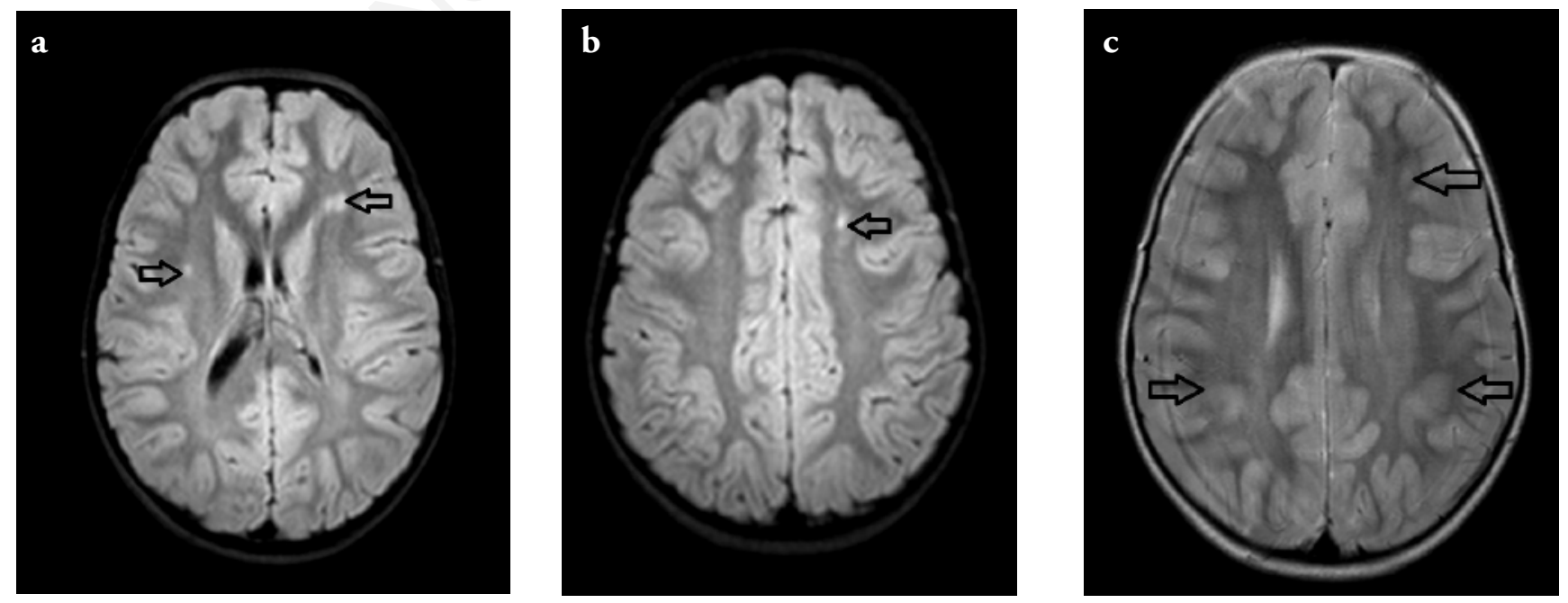

Figure 2. Changes in a patient, who received radiotherapy. Axial FLAIR image (a,b). Axial T2 image (c). Changes are shown by arrows. 
ple foci of leukoencephalopathy, MRappearance of which complied with multiple intracerebral microhemorrhages. Two years later, this patient developed isolated CNS relapse with damage localization in the optic disk. At relapse, no dynamics of leukoencephalopathy and microhemorrhages were found. The patient was excluded from the analysis owing to ambiguous interpretation of MR-phenomena.

\section{Neurocognitive tests}

By the time the tasks of screening tests were performed (for visual-motor coordination and understanding of instructions), both studied groups showed lower indices compared to the norm. During the passage of the main series of tests, which included tests for visual, spatial and working memory, as well as planning tests, statistically reliable results were obtained only on the working memory test: participants, who did not undergo irradiation, had more productive working memory, compared with participants who have undergone irradiation $(\mathrm{P}=0.095)$. When assessing the emotionalbehavioral sphere, the combined clinical group differed from the norm in almost all indicators $(\mathrm{P}=0.095)$. According to the results of the questionnaire of T.Achenbach, there was no difference between the groups $(\mathrm{P}<0.076)$. This picture indicates behavioral difficulties in children in the clinical group, regardless of the type of treatment. According to the M.Kovach children's depression questionnaire, no statistically significant differences between the groups were find.

In addition, there was no statistically based correlation between cognitive impairments and the degree of brain damage according to brain MRI $(\mathrm{P}<0.076)$.

\section{Discussion and Conclusions}

Analysis of brain MRI data in patients did not reveal significant differences in radiographic presentation of leukoencephalopathy between patients who received craniocerebral irradiation and who received additional intrathecal injections of chemotherapeutic

drugs. Leukoencephalopathy was found in about half of the patients. It was mostly diffuse and localized predominantly in the frontal and temporal lobes.

According to our data, the exclusion of radiation therapy from the treatment protocol of children with ALL did not have advantages over the use of additional intrathecal injections of cytotoxic drugs as prevention of leukemia from the point of view of changes on MRI. The analysis did not show significant differences in brain damage and severity of cognitive impairment depending on the type of prevention of CNS leukemia. Moreover, in this study, no statistical correlation was found between MRI leukoencephalopathy and neurocognitive impairment according to clinical tests data. At the time of the examination, patients were at different stages of rehabilitation, and the period after the end of therapy varied (from 9 days to 56 months). This detail is important because some patients may be at the stage of acute toxic changes, and some at the stage of late toxic effects. This study did not take into account such important effects of radiation therapy as the development of secondary tumors, neuroendocrine effects, the risk of vascular complications and late neurocognitive deficit. Perhaps the most promising is the use of modern methods for assessing brain activity and delicate brain structures (functional MRI, image diffusion tensor), which seems to be reasonable in this category of patients. Since the routine MRI performed even with at the scanner with an ultra-high magnetic field strength, does not provide information about possible damage at the level of pathways and functional centers. ${ }^{17}$ For example, innovative visualization of tensor diffusion (tractography), carried out in children before and after chemotherapy, revealed a decrease in conductivity through the internal capsule emitting the crown and the body of the colosseum. ${ }^{18}$

Thus, further comprehensive study of the late neurotoxic effects of radiation therapy and intrathecal chemotherapy is promising, given that the toxic effects of radiation and chemotherapeutic agents in addition to acute damage have long-term

Table 2. The results and analysis of brain MRI.

\begin{tabular}{|c|c|c|}
\hline & $\begin{array}{l}\text { Group with additional } \\
\text { lumbar punctions (\%) }\end{array}$ & $\begin{array}{l}\text { Group with cranial } \\
\text { irradiation (\%) }\end{array}$ \\
\hline Incidence of leukoencephalopathy & $17(66.6)$ & $10(50)$ \\
\hline $\begin{array}{l}\text { Size (\% out of all patients with lesions) } \\
\text { Only focal } \\
\text { Only diffuse } \\
\text { Combination of focal and diffuse lesions }\end{array}$ & $\begin{array}{l}12(70.6) \\
3(17.6) \\
2(11.8)\end{array}$ & $\begin{array}{c}8(80) \\
0 \\
2(20)\end{array}$ \\
\hline $\begin{array}{l}\text { Localization (\% out of all patients with lesions) } \\
\text { Frontal } \\
\text { Temporal } \\
\text { Parietal } \\
\text { Occipital }\end{array}$ & $\begin{array}{c}13(76.4) \\
6(35.3) \\
13(76.4) \\
9(52.9)\end{array}$ & $\begin{array}{l}8(80) \\
2(20) \\
9(90) \\
2(20)\end{array}$ \\
\hline $\begin{array}{l}\text { Spread (\% out of all patients with lesions) } \\
\text { One lobe } \\
\text { Two or more lobes }\end{array}$ & $\begin{array}{c}5(29.4) \\
12(70.6)\end{array}$ & $\begin{array}{l}3(30) \\
7(70)\end{array}$ \\
\hline
\end{tabular}
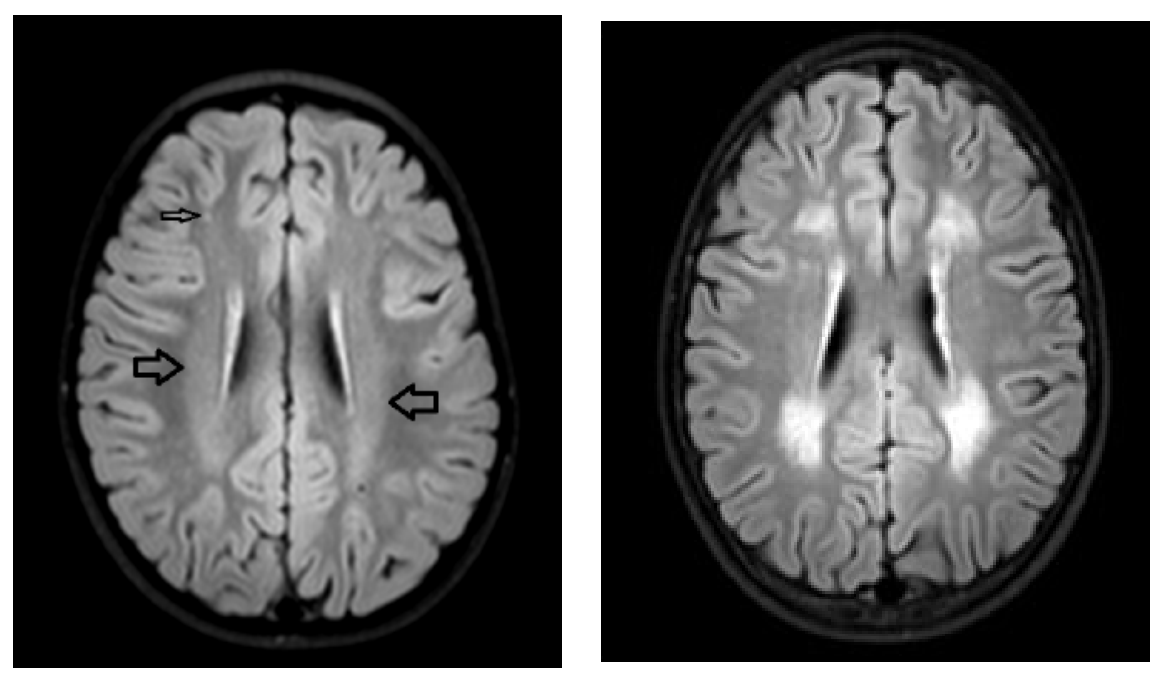

Figure 3. Changes in a patient, who received intrathecal chemotherapy. Axial FLAIR image. Changes are shown by arrows. 
effects. These toxic effects occur over time, so prospective studies with a long observation period are needed. Despite the lack of statistical differences in the toxicity of prophylactic methods of CNS leukemia in our study, there will be further development of effective methods for diagnostics and therapy of toxic brain damage in children. Patients from this study will be included in prospective studies, with repeated MRI using innovative methods and clinical examinations, including a full neurocognitive assessment.

\section{References}

1. Rumyantseva YV, Karachunsky AI, Aleynikova OV et al. Prognostic factors in a multicenter study of childhood acute lymphoblastic leukemia in children in Moscow-Berlin protocols. Oncohematol 2010;1:37-49. [Article in Russin].

2. Badr MA, Hassan TH, El-Gerby KM, Lamey ME. Magnetic resonance imaging of the brain in survivors of childhood acute lymphoblastic leukemia. Oncol Lett 2013;5:621-6.

3. Sharipova MG, Smirnov DS, Yakupov MR, Spichak II. Chemotherapy-induced polyneuropathy in children with oncological diseases. Pediatr Bull South Ural 2016;1:53-61.

4. Brugnoletti F, Morris EB, Laningham $\mathrm{FH}$, et al. Recurrent intrathecal methotrexate induced neurotoxicity in an adolescent with acute lymphoblastic leukemia: Serial clinical and radiologic findings. Pediatr Blood Cancer 2009;52:
293-5.

5. Tselovalnikova TYu, Pavlova MG, Zilov AV, et al. Growth disturbance after treatment for childhood acute lymphoblastic leukemia. Iss Oncol 2016;62:817-21.

6. Tamnes CK, Zeller B, Amlien IK, et al. Cortical surface area and thickness in adult survivors of pediatric acute lymphoblastic leukemia. Pediatr Blood Cancer 2015;62:1027-34.

7. Morioka S, Morimoto M, Yamada K, et al. Hosoi H. Effects of chemotherapy on the brain in childhood: diffusion tensor imaging of subtle white matter damage. Neuroradiology 2013;55:1251-7.

8. Daams M, Schuitema I, van Dijk BW, et al. Long-term effects of cranial irradiation and intrathecal chemotherapy in treatment of childhood leukemia: a MEG study of power spectrum and correlated cognitive dysfunction. BMC Neurol 2012;12:84.

9. Kuleva SA, Belogurova MB, Kolygin BA. Late neurotoxocity of antitumour treatment in children: Part 1. Oncologic Pediatr 2014;1:11-6.

10. Rumyantsev AG, Samochatova EV, Khamdan T. Acute lymphoblastic leukemia treatment in children according to the BFM programme. Journal «Pediatria» n.a. G.N. Speransky 1991;70:58-63. [in Russian].

11. Inaba H, Khan RB, Laningham, FH, et al. Clinical and radiological characteristics of methotrexate-induced acute encephalopathy in pediatric patients with cancer. Ann Oncol 2008;19:178.

12. Smirnov DS, Spichak II, Sharipova MG, Zhukovskaya EV. Polyneuropathy induced by chemotherapy in patients with acute lymphoblastic leukemia. Pediatr Bull South Ural 2015;2:66-9. [Article in Russian].

13. El-Fayech C, Haddy N, Allodji RS, et al. Cerebrovascular Diseases in Childhood Cancer Survivors: Role of the Radiation Dose to Willis Circle Arteries. Int J Radiation Oncol Biol Phys 2017;97:278-86.

14. Krawczuk-Rybak M, Grabowska A, Protas PT, et al. Intellectual functioning of childhood leukemia survivors--relation to Tau protein--a marker of white matter injury. Adv Med Sci 2012;57:266-72.

15. Reddick WE, Taghipour DJ, Glass JO, et al. Prognostic factors that increase the risk for reduced white matter volumes and deficits in attention and learning for survivors of childhood cancers. Pediatr Blood Cancer 2014;61:1074-9.

16. Kasatkin VN, Karachunsky AI, Malyh $\mathrm{SB}$, et al. Cognitive and behavioral characteristics of children with acute lymphoblastic leukemia who have completed treatment according to the MB2008 protocol: a pilot study. Iss Pract Pediatr 2015;10:7-13. [Article in Russian].

17. Nishikawa T, Okamoto Y, Maruyama S, et al. Acute encephalomyelitis complicated with severe neurological sequelae after intrathecal administration of methotrexate in a patient with acute lymphoblastic leukemia. Rinsho Ketsueki 2014;55:2306-10.

18. Hu Z, Zou D, Mai H, et al. Altered brain function in new onset childhood acute lymphoblastic leukemia before chemotherapy: A resting-state fMRI study. Brain Dev 2017;39:743-50. 